\title{
Matter wave soliton collisions in the quasi one dimensional potential
}

\author{
Nguyen Viet Hung, ${ }^{1}$ Michał Matuszewski, ${ }^{2}$ and Marek Trippenbach ${ }^{1,3}$ \\ ${ }^{1}$ Soltan Institute for Nuclear Studies, Hoża 69, PL-00-681 Warsaw, Poland \\ ${ }^{2}$ Nonlinear Physics Center and ARC Center of Excellence for Quantum Atom Optics, \\ Research School of Physical Sciences and Engineering, \\ Australian National University, Canberra ACT 0200, Australia \\ ${ }^{3}$ Institute for Theoretical Physics, Warsaw University, Hoża 69, PL-00-681 Warsaw
}

\begin{abstract}
We consider soliton solutions of a two-dimensional nonlinear system with the self-focusing nonlinearity and a quasi-1D confining potential, taking harmonic potential as an example. We investigate a single soliton in detail and find criterion for possible collapse. This information is then used to investigate the dynamics of the two soliton collision. In this dynamics we identify three regimes according to the relation between nonlinear interaction and the excitation energy: elastic collision, excitation and collapse regime. We show that surprisingly accurate predictions can be obtained from variational analysis.
\end{abstract}

PACS numbers: 03.75.Lm, 05.45.Yv, 42.65.Tg

\section{INTRODUCTION}

Solitons are universal entities in the nonlinear science and interactions between them are perhaps the most fascinating features. Understanding soliton collisions is both of fundamental interest and of importance to its wealth and proposed applications. If the system is integrable, the collisions are elastic [1]. An example of such a system is $1 \mathrm{D}$ soliton in attractive nonlinear Kerr medium. However, even if we weakly perturb nonlinear Schrödinger equation, we can observe a chaotic character of two-soliton collisions [2, 3]. In the general case, it is necessary to resort to detailed numerical calculations for predictions. Stegeman and Segev [4] have introduced a general classification of collisions into two categories: coherent and incoherent. Coherent interactions occur when the nonlinear medium can respond to interference effects that take place when the beams overlap. Incoherent interactions, on the other hand, occur when the relative phase between the soliton varies much faster than the response time of the medium [5]. In Ref. [4] and for example in Ref. [6] one can find description of spatial soliton collisions in the Kerr medium. Collisions between spatiotemporal solitons of different dimensionality in a planar waveguide were considered in [7]. Here we also study collisions in the Kerr medium, which has infinitely short response time, but we are dealing with a pair of two dimensional solitons, both of the same dimensionality. We investigate head on collision of solitons moving in quasi 1D confining potential. Our numerical results were obtained for the harmonic potential, but they generally apply to all quasi $1 \mathrm{D}$ confining potentials that posses at least one bound state. We would like to point out that multidimensional solitons were extensively studied in the case of Bose Einstein condensates, including beautiful experiments [8]. Some of the applications of solitons in the condensates was discussed in Ref. [9].

Within the framework of current publication the statics and the dynamics of solitons in quasi 1D potentials are described within the variational approximation and compared with full numerical simulations. We find formulas for the widths and chemical potential and discuss quasi 1D limit and the dynamics of the collapse. Similar considerations were presented by Salasnich et al in reference [10] in the case of Bose-Einstein condensate [11, 12] and earlier for the optical beam propagation by Li et al 13]. Analogous study of the stability of gap solitons were presented in [14]. Finally we address the problem of collisions, in various regimes, from elastic collision regime, through the domain where the transverse excitations occur, up to the collapse during the collision. The main result of our study is the analysis of the collapse during the collision. We find it somehow surprising how good estimate can obtained from a simple variational model. We would like to point out that the deviation from one dimensionality in stationary properties and collisional dynamics of matter-wave solitons was recently investigated in [15] using an effective one-dimensional GrossPitaevskii equation that includes an additional quintic self-focusing term. 2D soliton collisions were also studied experimentally in the case of spin wave envelope solitons in Ref. [16].

\section{QUASI-1D APPROXIMATION}

In this paper we consider two dimensional system with attractive nonlinearity, with an additional external quasi$1 \mathrm{D}$ potential. This system is described by $2 \mathrm{D}$ nonlinear Schrödinger equation (NLSE)

$$
i \Psi_{t}=-\frac{1}{2}\left(\Psi_{x x}+\Psi_{y y}\right)+V \Psi-\lambda_{2 D}|\Psi|^{2} \Psi
$$

where $\lambda_{2 D}>0$. We assume that the wavefunction is normalized to $N$. All the calculations were performed for the specific case quasi $1 \mathrm{D}$ harmonic confining potential $V(x, y)=\omega x^{2} / 2$, see Fig. (11). Our predictions and physical properties derived here apply all the systems, two- and three dimensional, which have an external potential, confining in all but one dimension (in literature 


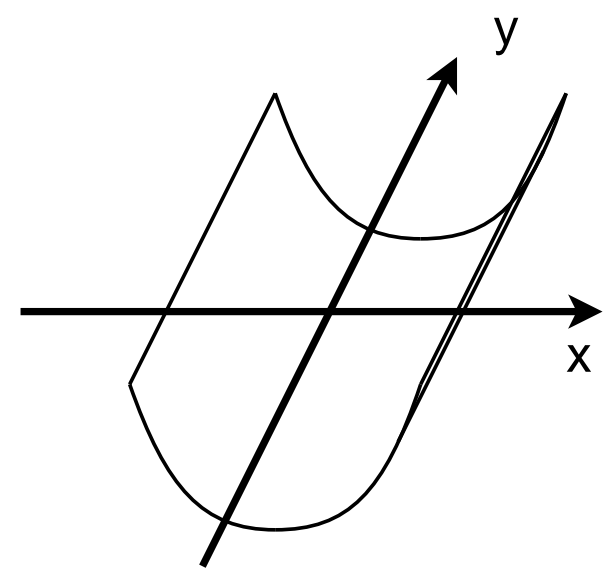

FIG. 1: Quasi 1D potential with harmonic confinement.

they are sometimes called "potentials with transverse confinement") that support at least one bound state in the transverse direction. From the existing literature on that subject we would like to acknowledge three examples that are closely related to our study: 1)Li et al [13] considered the simple variational model of spatial solitons in planar waveguides, 2) The existence of 3D solitons in the inhomogeneous medium with harmonic potential was investigated by Raghavan [17], 3) The dynamics of systems under transverse confinement were studied in BEC by Salasnich et al [10].

In the case of BEC, the equation (11) can be derived from the full three-dimensional Gross-Pitaevskii equation for the wavefunction in physical coordinates $\tilde{\Psi}(\tilde{\mathbf{r}}, \tilde{\mathbf{t}})$

$$
i \hbar \tilde{\Psi}_{\tilde{t}}=-\frac{\hbar^{2}}{2 m} \tilde{\nabla}^{2} \tilde{\Psi}+U(\tilde{\mathbf{r}}) \tilde{\Psi}-\lambda_{3 D}|\tilde{\Psi}|^{2} \tilde{\Psi},
$$

where $\lambda_{3 D}=-4 \pi a_{s} \hbar^{2} / m, m$ is the atomic mass, wavefunction norm $N$ is the number of atoms, and $a_{s}$ is the scattering length. By assuming strong harmonic confinement $\omega_{z}$ in the $z$ direction, $U(\tilde{\mathbf{r}})=m \omega_{z}^{2} \tilde{z}^{2} / 2+$ $U_{\perp}(\tilde{x}, \tilde{y})$, that prevents excitation of higher modes of the trap in this direction, we arrive in dimensionless Eq. (11) after performing rescaling according to $(x, y)=$ $(\tilde{x}, \tilde{y}) / x_{0}, t=\left(\hbar / m x_{0}^{2}\right) \tilde{t}, \quad V=\left(m x_{0}^{2} / \hbar^{2}\right) U, \lambda_{2 D}=$ $\left(m / \hbar^{2}\right) \sqrt{m \omega_{z} / 2 \pi \hbar} \lambda_{3 D}$ and $\Psi=x_{0} \tilde{\Psi}$. Here $x_{0}$ is an arbitrary scaling parameter.

In all the nonlinear systems with quasi 1D potentials, as long as the transverse part of the potential supports at least one bound state, we expect, in certain range of the strength of the nonlinearity, to find 2D soliton solutions. More precisely, in such systems for small values of nonlinearity we find stable solitons, but there is a threshold, a critical value of nonlinearity at which catastrophic selffocusing occurs. When we approach this critical value our soliton turns into the Townes soliton [18], and above this value no stable solitons are available.

As we mentioned above, to focus our attention in what follows we concentrate on the model with harmonic confinement. In the limit when the trapping frequency be- comes large we expect that the energy associated with the nonlinear interaction becomes negligible in comparison with $\hbar \omega$. In this case our 2D soliton will become a product of a ground state in the direction of the confining potential and 1D soliton in an unbound direction. The following calculation, based on the separation of variables, confirms this statement.

If we assume that our wavefunction $\Psi(x, y, t)$ can be presented as a product of

$$
\Psi(x, y, t)=\phi(x) \tilde{\psi}(y, t),
$$

where $\phi(x)$ is normalized to unity, we can rewrite equation (1)

$$
\begin{aligned}
& {[\phi(x) \tilde{\psi}(y, t)]_{t}=\left[-\frac{1}{2} \phi_{x x}(x)+\frac{1}{2} \omega^{2} x^{2} \phi(x)\right] \tilde{\psi}(y, t)} \\
& -\left[\frac{1}{2} \tilde{\psi}_{y y}(y, t)+\lambda_{2 D}|\tilde{\psi}(y, t)|^{2} \tilde{\psi}(y, t)|\phi(x)|^{2}\right] \phi(x),
\end{aligned}
$$

and if we assume that $\phi(x)$ is a ground state of the trapping potential we obtain

$$
\left[\tilde{\psi}_{t}-\frac{1}{2} \omega^{2} \tilde{\psi}\right] \phi=-\frac{1}{2} \tilde{\psi}_{y y} \phi+\lambda_{2 D}|\tilde{\psi}|^{2} \tilde{\psi}|\phi|^{2} \phi .
$$

Upon multiplying both sides of the equation by $\phi^{*}(x)$, integrating over $x$ and neglecting constant term we obtain one dimensional (NLSE) for $\psi=\tilde{\psi} \exp \left(-\omega^{2} t / 2\right)$ (see Eq. (77) below), with effective nonlinearity equal to

$$
\lambda_{1 D}=\lambda_{2 D} \int d x|\phi(x)|^{4} .
$$

We will call this regime a quasi $1 \mathrm{D}$ limit. Notice, that in the case of harmonic potential $\int d x|\phi(x)|^{4}=\sqrt{\omega /(2 \pi)}$.

One dimensional NLSE is fully integrable. Consequently, we expect that two solitons, that are coming into collision will asymptotically reassume their original shape after collision. If the nonlinear energy becomes comparable with the excitation energy of the confining potential, we expect to see growing deviation from 1D dynamics. It is the main purpose of this presentation to study this deviation.

\section{VARIATIONAL APPROXIMATION}

\section{A. 1D soliton}

The nonlinear Schrödinger equation in 1D case reads

$$
i \psi_{t}=-\frac{1}{2} \psi_{y y}-\lambda_{1 D}|\psi|^{2} \psi,
$$

where we assume that norm of the wavefunction is equal to $\int_{-\infty}^{\infty}|\psi|^{2} d y=N$. Within the variational approximation [19, 20], instead of solving Eq. (7) we introduce Lagrange density

$$
\mathcal{L}=\frac{1}{2}\left[i\left(\psi_{t} \psi^{*}-\psi_{t}^{*} \psi\right)-\left|\psi_{y}\right|^{2}+\lambda_{1 D}|\psi|^{4}\right] .
$$


To mimic the dynamics we will introduce a trial function of the form (variational Ansatz)

$$
\psi(y, t)=A(t) \exp \left(-\frac{y^{2}}{2}\left[\frac{1}{V(t)^{2}}-i b(t)\right]+i \phi(t)\right),
$$

with variational parameters: amplitude $A(t)$, chirp $b(t)$, overall phase $\phi(t)$ and width $V(t)$. By substituting Ansatz (9) into our Lagrange density and integrating over $y$ we obtain the Lagrange function $L=$ $\sqrt{\pi} A^{2}\left[-\dot{\phi} V-\frac{\dot{b} V^{3}}{4}-\frac{1}{4 V}-\frac{b^{2} V^{3}}{4}+\frac{\lambda_{1 D} A^{2} V}{2 \sqrt{2}}\right]$. Our variational approximation restricts the set of available solutions to the certain class of functions. It will lead to the Euler - Lagrange equations of the reduced Lagrangian shown above

$$
\begin{aligned}
\dot{\phi} & =\frac{\lambda_{1 D} A^{2}}{\sqrt{2}}-\frac{1}{4 V^{2}}-\frac{V \ddot{V}}{4}, \\
\dot{V} & =\frac{1}{V^{3}}-\frac{\lambda_{1 D}}{\sqrt{2 \pi} V^{2}} \text { and } b=\frac{\dot{V}}{V}
\end{aligned}
$$

with additional one, which we can interpret as a conservation law (first integral) $A^{2} V=$ const, and which is related to the norm of the trial function

$$
\int_{-\infty}^{\infty}|\psi|^{2} d y=A^{2} V \sqrt{\pi}=N \Rightarrow A=\sqrt{\frac{N}{V \sqrt{\pi}}} .
$$

In this formalism solitons correspond to stationary solutions of Eq. (10) i. e. we assume $\dot{V}=\ddot{V}=0$. These conditions can be satisfied when $V=\frac{\sqrt{2 \pi}}{N \lambda_{1 D}}$ and $b=0$. Notice that we can evaluate soliton eigenvalue. In nonlinear optics this eigenvalue corresponds to the soliton wavevector and in the theory of BEC it becomes chemical potential. We write the solution in the form

$$
\psi(y, t)=\Phi(y) \exp \left(-i \mu_{1 D} t\right) .
$$

To evaluate $\mu_{1 D}$ we substitute $V$ into the equation for phase, and obtain

$$
\dot{\phi}=-\mu_{1 D}=-\frac{3 \lambda_{1 D}^{2} N^{2}}{8 \pi} .
$$

\section{B. 2D soliton}

In this case we start with 2D nonlinear Schrödinger equation with harmonic potential

$$
i \Psi_{t}=-\frac{1}{2}\left(\Psi_{x x}+\Psi_{y y}\right)+\frac{1}{2} \omega^{2} x^{2} \Psi-\lambda_{2 D}|\Psi|^{2} \Psi,
$$

with normalization $\iint_{-\infty}^{\infty}|\Psi|^{2} d x d y=N$. The 2D Lagrange density is

$$
\begin{aligned}
\mathcal{L}= & \frac{1}{2}\left[i\left(\Psi_{t} \Psi^{*}-\Psi_{t}^{*} \Psi\right)-\left|\Psi_{x}\right|^{2}-\right. \\
& \left.-\left|\Psi_{y}\right|^{2}-\omega^{2} x^{2}|\Psi|^{2}+\lambda_{2 D}|\Psi|^{4}\right] .
\end{aligned}
$$

We use 2D Gaussian Ansatz

$$
\begin{aligned}
\Psi(x, y, t)= & A(t) \exp \left(-\frac{x^{2}}{2 W(t)^{2}}-\frac{y^{2}}{2 V(t)^{2}}\right) \times \\
& \times \exp \left(i\left[\phi(t)+\frac{1}{2}\left(b(t) x^{2}+c(t) y^{2}\right)\right]\right),
\end{aligned}
$$

with variational parameters $A(t), \phi(t), b(t), c(t), W(t)$ and $V(t)$. In analogy with what we presented above we obtain (after integrating over $x$ and $y$ ) the Lagrangian and look for the stationary solutions $(\dot{W}=\ddot{W}=0$ and $\dot{V}=\ddot{V}=0$ ), which occur when the following conditions are satisfied: $c=0, b=0$ and

$$
V=\frac{2 \pi}{\lambda_{2 D} N} W, \text { and } W=\sqrt[4]{\frac{4 \pi^{2}-\lambda_{2 D}^{2} N^{2}}{4 \pi^{2} \omega^{2}}}
$$

First significant observation is the clear evidence of collapse in our model. Note that equation for $W$ can not be satisfied when $\lambda_{2 D} N \geq 2 \pi$. Close to this critical point, both widths become equal and tend to zero.

To obtain the value of the chemical potential we substitute the width obtained in Eq. (16) into equation for $\dot{\phi}$ and get

$$
\dot{\phi} \equiv-\mu_{2 D}=-\frac{\omega}{2 \pi} \frac{2 \pi^{2}-\lambda_{2 D}^{2} N^{2}}{\sqrt{4 \pi^{2}-\lambda_{2 D}^{2} N^{2}}}=-\frac{\omega}{2} \frac{1-2 \eta^{2}}{\sqrt{1-\eta^{2}}},
$$

where $\eta=\left(\lambda_{2 D} N\right) /(2 \pi)$. In the analogy with 1D case we can write $\Psi(x, y, t)=e^{-i \mu_{2 D} t} \Phi(x, y)$, Similar variational approach can be developed for the case of the quasi 1D square well potential. The only difference in the functional form of the effective Lagrangian, and therefore also in the equations of motion, would be in potential term, which contains $\omega$, Finally we would like to point out that the analysis presented above can be used in the linear limit, when the waveguide mode structure can be predicted with satisfactory accuracy, see [13].

\section{Collapse of the 2D wavefunction}

We now consider the case when $\eta \geq 1$, i. e. in the regime where we expect the wavefunction to collapse. As we mentioned above, close to the collapse both widths of our solution, $W$ and $V$ become even. Hence, in the crude approximation, to describe the dynamics of the collapse we can assume axial symmetry. Upon neglecting harmonic potential contribution (which is negligible during the collapse) we obtain

$$
\ddot{W}=\frac{1}{W^{3}}-\frac{\eta}{W^{3}}=\frac{1-\eta}{W^{3}} .
$$

If we solve the Eq. (18) with initial conditions $W(0)=$ $W_{0}, \dot{W}(0)=0$, which correspond to the dynamics originated from some unstable state, we obtain

$$
W(t)=W_{0} \sqrt{1-\frac{(\eta-1) t^{2}}{W_{0}^{4}}}
$$



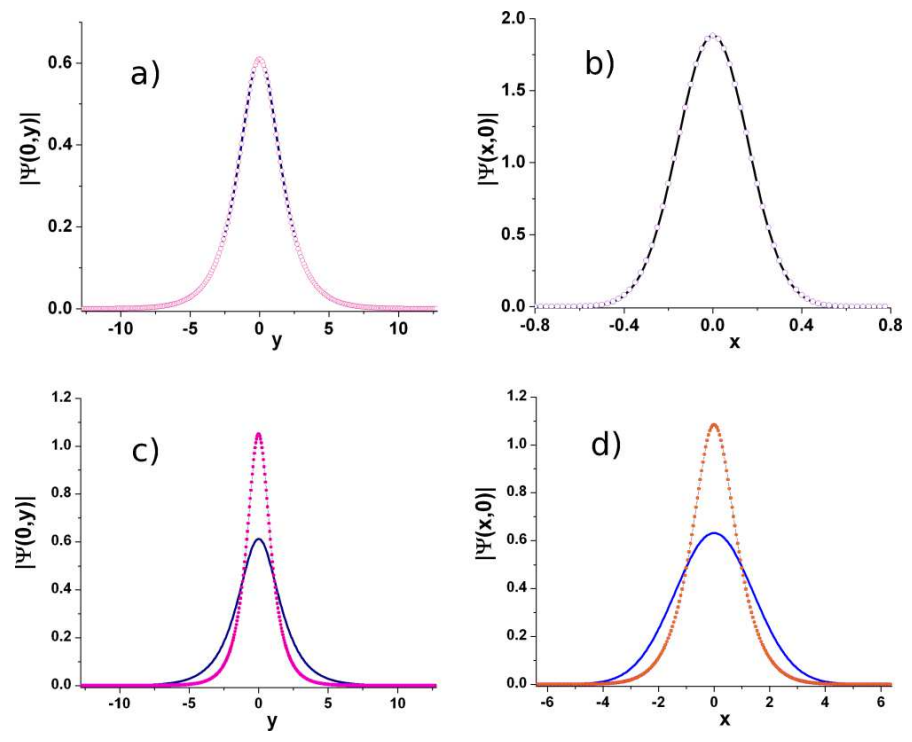

FIG. 2: Comparison of the 2D soliton cross sections (dots) (in $x$ and $y$ planes) with quasi $1 \mathrm{D}$ approximation (continuous curves). a) and b) corresponds to the frequency $\omega=40$. We see a perfect agreement between 1D soliton and cross section of $2 \mathrm{D}$ soliton along $y$ axis and ground state of the harmonic potential and $2 \mathrm{D}$ soliton cross section along $x$ axis. c) and d) were obtained for smaller frequency $\omega=0.5$. Some deviation from quasi $1 \mathrm{D}$ approximation can be observed.

The main conclusion from this simple calculation is that the collapse of the wavefunction occurs within the finite time (except when $\eta=1$, on the threshold for the collapse). The collapse time is equal to

$$
t_{c o l}=\frac{W_{0}^{2}}{\sqrt{\eta-1}}
$$

Collapse occurs on the timescale that is proportional to $W_{0}^{2}$, but what is more important it occurs the faster the the higher norm of the wavefunction is. This will be important in the next section when we discuss collapse during the soliton collision.

To relate our result to experiments, we calculate the critical atom number necessary to observe collapse in BEC. We consider a ${ }^{85} \mathrm{Rb}$ condensate in a highly anisotropic trap configuration with $\omega_{z}=2 \pi \times 350 \mathrm{~Hz}$, $\omega_{y}=2 \pi \times 55 \mathrm{~Hz}$, and a shallow confinement in the $x$ direction. This configuration can be realized using an optical dipole trap 21]. For the scattering length value of $a_{s}=-15 a_{0}$ 12] the critical number of atoms is $N_{\mathrm{cr}} \approx 10^{3}$, and the typical soliton dimensions close to the collapse threshold are of the order of several $\mu \mathrm{m}$.

\section{NUMERICAL RESULTS}

\section{A. Quasi-1D limit}

We now consider the case when value of $\lambda_{2 D}$ (see Eq. (6)) tends to zero, but the value of $\lambda_{1 D}$ and the

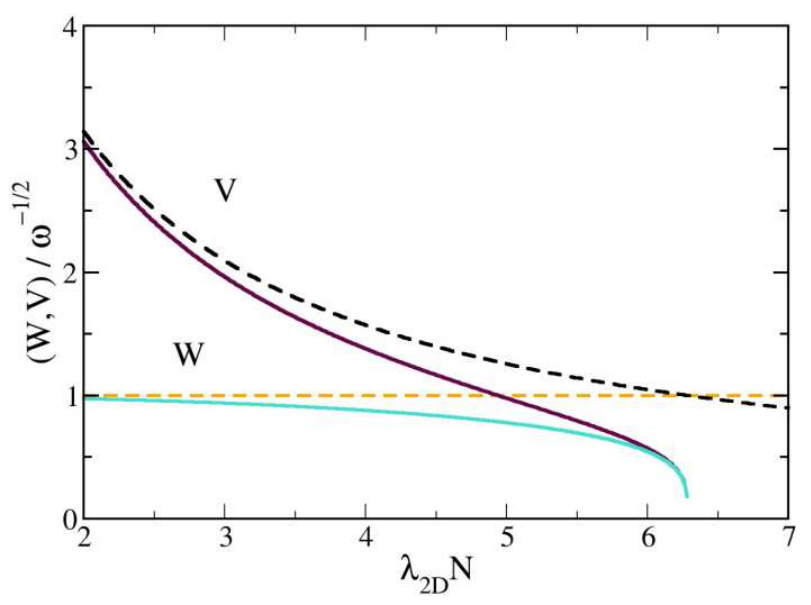

FIG. 3: Widths of the 2D soliton obtained from variational approximation, as a function of parameter $\lambda_{2 D} N$ (solid lines). Marked with dashed line are the corresponding values of $1 \mathrm{D}$ soliton width and the width of the ground state of the harmonic 1D potential (horizontal line).

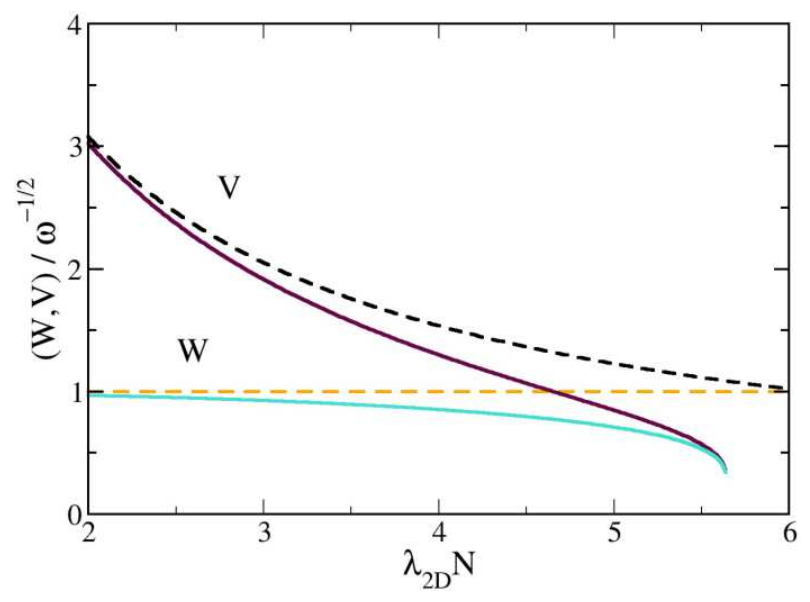

FIG. 4: Widths of the 2D soliton obtained from direct numerical simulations, as a function of nonlinear parameter $\lambda_{2 D} N$.

norm remain constant. This corresponds to the limit $\omega \rightarrow \infty$. By substituting 2D nonlinear coefficient $\lambda_{2 D}=$ $\lambda_{1 D} \sqrt{2 \pi / \omega}$ into the expression for $2 \mathrm{D}$ eigenvalue $\mu_{2 D}$ in Eq. (17), we obtain

$$
\mu_{2 D} \simeq \frac{\omega}{2}-\frac{3 \lambda_{1 D}^{2} N^{2}}{8 \pi}=\frac{\omega}{2}+\mu_{1 D} .
$$

This way we show that 2D eigenvalue, in the limit of high frequency, consists of 1D eigenvalue and the ground state energy of harmonic oscillator. It is a very intuitive result, since in this limit 2D soliton becomes a product state of 1D soliton and the ground state of the harmonic potential in the transverse direction. Now we examine the widths of $2 \mathrm{D}$ soliton presented in Eq. (16). If we assume $\omega \rightarrow \infty$ one of the widths $W \simeq 1 / \sqrt{\omega}$, and $V \rightarrow \sqrt{2 \pi} /\left(\lambda_{1 D} N\right)$. This is exactly the value we obtained for the $1 \mathrm{D}$ soliton. In conclusion, in the limit considered here, both 


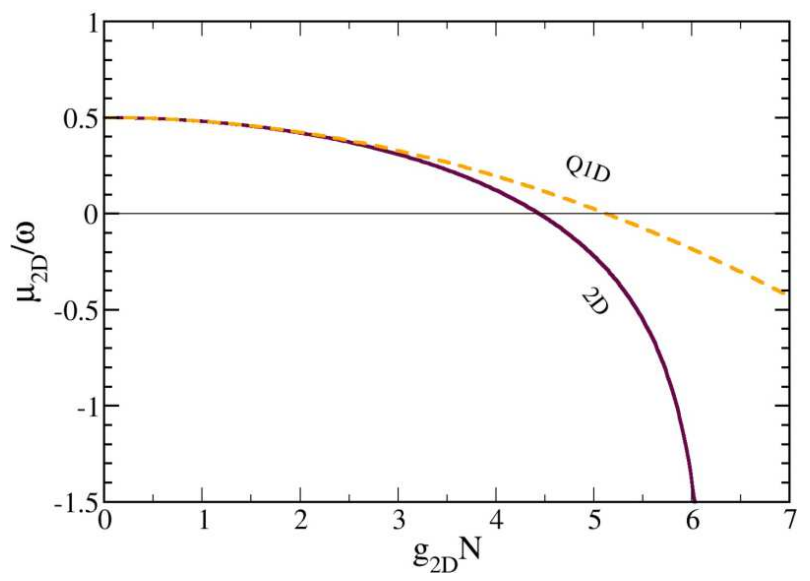

FIG. 5: Comparison of the chemical potential $\mu_{2 D}$ obtained using quasi 1D approximation (Eq. (21) (dashed line) and full 2D variational approximation (Eq. (17) (solid line) as a function of nonlinear parameter $\lambda_{2 D} N$. Notice that the region between the collapse and the point up to which quasi $1 D$ approximation holds is very narrow.

chemical potential and the width obtained from variational approximation take proper quasi $1 \mathrm{D}$ values. In Fig. 2 we present both cross-sections of $2 \mathrm{D}$ soliton in $x$ and $y$ planes. One of the cross sections is compared with 1D soliton and the other with the ground state of the harmonic potential. The comparison is made for two different values of frequency $\omega$. For larger value of $\omega$ we observe perfect matching and when $\omega$ becomes smaller, we can see slight deviations from quasi 1D approximation. In Fig. 3] we present 2D soliton width obtained from variational approximation, as a function of parameter $\lambda_{2 D} N$. For comparison we also included a dashed line representing a width of the 1D soliton, and straight dashes horizontal line corresponding to the width of the ground state of the trapping potential (Gaussian). In Fig. 4 the same comparison is made for the case of numerical solutions. In this case we defined the widths numerically, as $(V, W)=\sqrt{\pi}(\langle|x|\rangle,\langle|y|\rangle)$, i. e. the the mean value of the modulus of the coordinate in this direction. We clearly see that for small $\lambda_{2 D} N 2 \mathrm{D}$ soliton width well approximates the equivalent $1 \mathrm{D}$ value, and when $\lambda_{2 D} N$ approaches critical value, both widths become equal, while tending to zero. In this limit, the energy of the trapping potential is much smaller than kinetic and nonlinear energies, hence our solution becomes practically identical as that of Townes soliton [18]. Finally, in Fig. 5 we plot a chemical potential as a function of $\lambda_{2 D} N$ obtained within quasi 1D approximation (Eq. (21)) and that obtained from full 2D variational analysis, (formula (17)). Notice that when the $\mu_{2 D}$ is of order of the excitation energy in the harmonic potential quasi 1D approximation breaks down. Also on this figure we can see a clear indication of the collapse region, when $\lambda_{2 D} N$ approaches the value of $2 \pi$, and the fact that region between the collapse and the point up to which quasi 1D approximation holds

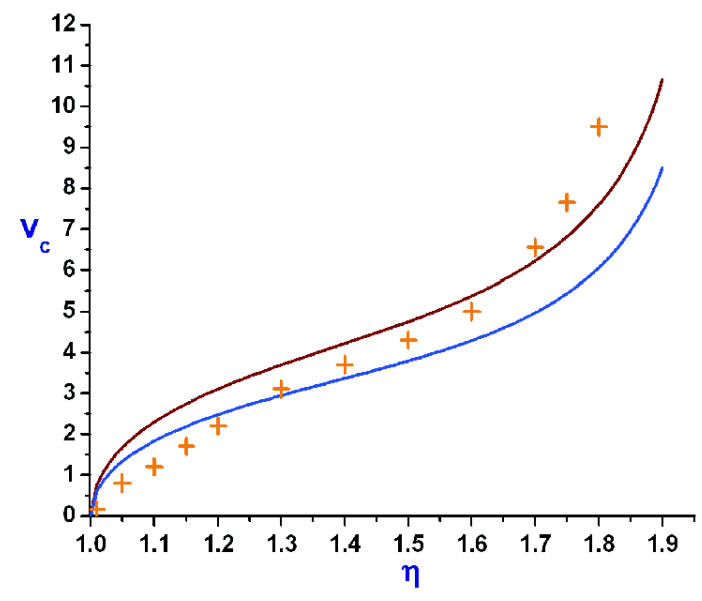

FIG. 6: Boundaries of the collisional collapse region for the trap with the frequency $\omega=4$, obtained from variational approximation (with Gaussian - upper continuous curve and hyperbolic secant trial functions - lower continuous curve) and obtained from direct numerical simulations (crosses). Solitons colliding with velocities below those marked with crosses will experience collapse during their interaction.

is very narrow.

\section{B. Soliton collisions}

Now we investigate collision of solitons that are moving along the quasi 1D harmonic potential presented in Fig. 1. Soliton collisions in the one-dimensional NLSE were studied in many contexts, including nonintegrable dynamics of vector solitons [22]. In the case of BEC, the quasi-1D collisions were investigated in Ref. [10] in the framework of the nonpolynomial Schrödinger equation (NPSE). The results obtained here can be easily generalized for the quasi 1D square well potential. The solitons that we used in the simulations were identical, and had equal and opposite velocities.

We start with a pair of solitons, each of which is separately a solution of Eq. (1). The initial width along free direction we denote by $V_{0}$ and the in the transverse direction by $W_{0}$. We assume that the norm of each soliton as equal to $N / 2$ and they travel with velocity $v$. The nonlinear interaction is described by the parameter $\lambda_{2 D}$. We will introduce here a simple estimate of collapse during the collision based on variational approximation. It is well known that estimates based on variational analysis depends on the choice of variational basis, up to the multiplicative factor [23]. Hence, to improve the quality of our predictions we will consider the estimates obtained using Gaussian functions and hyperbolic secant functions. Lets first concentrate on Gaussians. The first parameter that describes collision is soliton interaction 
time. This is merely the time when both wavefunctions have a significant overlap. If we assume that significant interaction appear as long as wavepackets are separated not more than FWHM, we obtain interaction time equal to

$$
t_{i n t} \simeq \frac{2 \gamma V_{0}}{v}=\frac{4 \gamma W_{0}}{\eta v},
$$

where $\gamma \simeq 1.178$. Notice that the width ratio differs from the one given in Eq. (16), because soliton norm is now $N / 2$ instead of $N$. Here we also assume that during this time interaction is almost constant and is taken as that of full overlap (both function on top of each other). Now we turn to the condition for the collapse. As we see from Sec. IIIC collapse is expected to occur for $\eta>1$. This condition, in the case of collision, should refer to the situation when wavepackets fully overlap. On the other hand soliton with the norm $N / 2$ can only exist if $\eta<2$. Taking both conditions into account we conclude that the region of interest is $1<\eta<2$. We estimate a time of collapse for solitons in the above interval to be

$$
t_{c o l}=\frac{W_{0}^{2}}{\sqrt{\eta-1}} .
$$

Notice that here $W_{0}$ is the initial width of each of the colliding partners and given by

$$
W_{0}=\sqrt[4]{\frac{4-\eta^{2}}{4 \omega^{2}}}
$$

From the above discussion it follows that there should be a critical velocity, above which during the collision the collapse will take place. This condition can be obtained by comparing time of collision and time of collapse

$$
\frac{4 \gamma W_{0}}{\eta v_{c}} \simeq \frac{W_{0}^{2}}{\sqrt{\eta-1}},
$$

which after some algebra will give

$$
v_{c}(\eta)=\frac{4 \gamma \sqrt{2 \omega}}{\eta} \sqrt[4]{\frac{(\eta-1)^{2}}{4-\eta^{2}}} .
$$

If the velocity of solitons exceeds this critical value, there is not enough time during the collision to complete the collapse. If the velocity is lower than this value, during the overlap time collapse can fully develop. Hence the function $v_{c}(\eta)$ marks the boundary between regions of collapse and no collapse.

As we mentioned above, position of this line depends on the choice of the trial functions, which in the case just described were Gaussians. Had we chosen hyperbolic secant functions instead of Gaussians, we would have obtained slightly different result. First, we find that in this case the collapse for single soliton appear for the value of nonlinearity $\left(\lambda_{2 D} N\right)$ equal to 6 instead of $2 \pi$. Hence, the parameter $\eta$ should be defined as $\left(\lambda_{2 D} N\right) / 6$, and after algebra, very similarly to what we presented for Gaussians, we obtain the condition for the critical velocity as

$$
v_{c}(\eta)=\frac{8 \gamma \sqrt{\omega}}{\sqrt{\pi} \eta} \sqrt[4]{\frac{(\eta-1)^{2}}{4-\eta^{2}}}
$$

In Fig. 6 we show curves representing $v_{c}$ as a function of $\lambda_{2 D} N$, obtained from Eqs. (26) and (27), indicated with solid lines, together with the results obtained from direct numerical simulations (crosses). The agreement between the variational predictions and direct numerical simulations is very good. It is somehow surprising. It was shown in Reference 24] that the variational analysis of the collapse dynamics does not work very well close to the threshold. Nevertheless it seems that for our crude estimate it is sufficient. All the crosses are falling between curves obtained with Gaussian and hyperbolic secant trial functions. Solitons colliding with velocities below those marked with crosses will experience collapse during their interaction. In conclusion, we see that variational analysis predicts correctly and accurately the onset of catastrophic collapse during soliton collision.

The full picture of the soliton collision that emerges from our numerical studies is the following. Imagine we keep the norm of each soliton and their velocity constant. Then, depending on the strength of the confining potential ( $\omega$ in the case of harmonic potential and the width in the case of quasi 1D square well potential) we can clearly identify three regimes according to the relation between nonlinear interaction and the excitation energy. In the first regime, when the distance between ground state and first excited state of the confining potential is much bigger than the interaction energy, system is practically integrable, hence solitons pass though each other and restore their original shape after the collision. When both energy scales become comparable we observe excitations in the transverse directions, corresponding to the transition between ground state and first coupled excited state. Finally, we observe catastrophic collapse during the collision. Behavior of solitons in these three regions is illustrated in Fig. 7 Picture a) is characteristic for the first region described above. It was obtained for high frequency $\omega=20$ and small nonlinearity $\lambda_{2 D}=2$. Solitons appear to be practically one dimensional, and they asymptotically (after the collision) restore their original shape. In the case b) $\omega=2$ and nonlinearity is the same as in previous case and nonlinear interaction becomes comparable with excitation energy. Some of the population will be transferred to the excited state and we observe the beats of the frequency $2 \omega$ in the transverse direction. It can be detected for example by calculating mean square radius in the transverse direction, shown in the inset on the right hand side. Finally, in picture c) where $\lambda_{2 D}=3$ and $\omega=2$, total population is above the critical value and collision time is sufficiently long we observe collapse during the collision. Analogous effect was previously observed in optics, when two spatial 

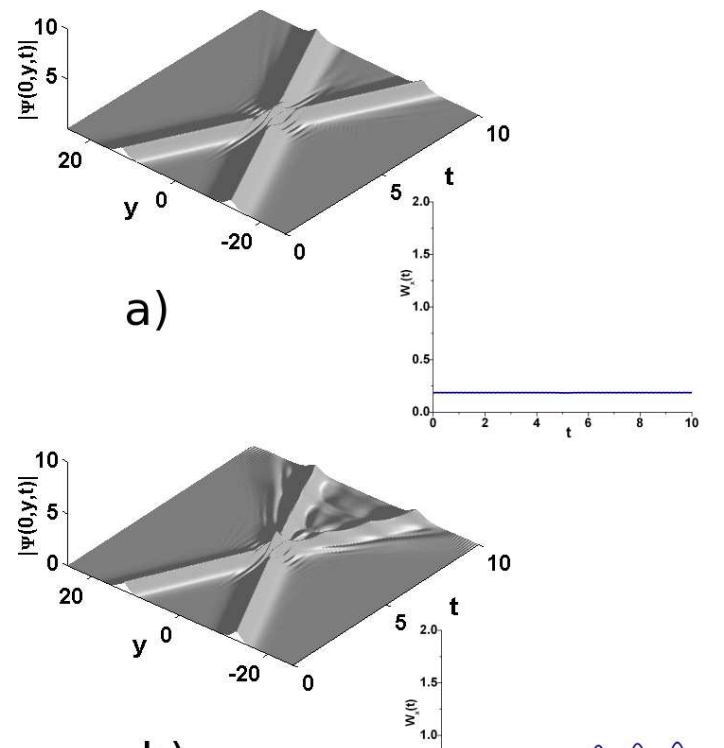

b)

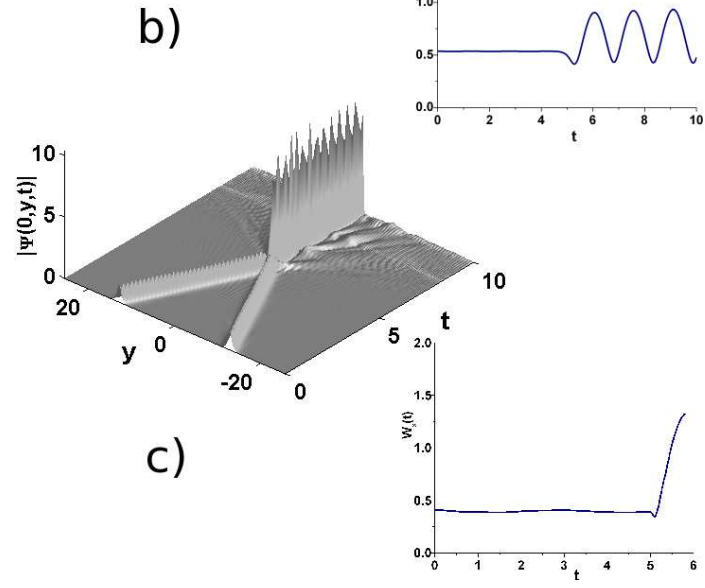

FIG. 7: Gallery of collisions. From quasi 1D to collapse. Soliton velocity $v=2.5$. Panel a) high frequency $(\omega=20)$ and small nonlinearity $\lambda_{2 D}=2$ - solitons appear to be practically one dimensional, and they asymptotically restore their original shape. Panel b) small frequency $\omega=2$ and nonlinearity the same as in previous case - nonlinear interaction is comparable with excitation energy. We observe oscillations with frequency $2 \omega$ (inset on the right hand side shows mean square radius in the transverse direction as a function of time). Panel c) frequency the same as in case b), but higher nonlinearity $\lambda_{2 D}=3$ - we observe collapse during the collision. solitons collided in planar waveguides, see for instance [16].

\section{CONCLUSIONS}

In conclusion, we analyzed the stability and collisions of quasi $1 \mathrm{D}$ solitons in the confining potential, both numerically and within variational approximation. We showed that variational approximation constitutes an excellent basis for estimating physical parameters of quasi $1 \mathrm{D}$ solitons. The advantage of using this approximation is that one obtains analytical formulas, and can predict soliton behavior in different limits and under various circumstances. One of the examples is the threshold of the collapse and its dynamics. Comparing time of collapse and collision time we could predict the result of the soliton collision and identify three different regimes according to the relation between nonlinear interaction and the excitation energy. In the first regime, when the distance between ground state and first excited state of the confining potential is much bigger than the nonlinear interaction, system is practically integrable, hence solitons pass through each other and restore their original shape after the collision. When both energy scales become comparable we observe excitations in the transverse directions, corresponding to the transition between ground state and first coupled excited state. Finally we observe catastrophic collapse during the collision. In the following paper we will present similar considerations for the case of Gap solitons.

\section{ACKNOWLEDGEMENTS}

M.T. would like to thank Prof. Yuri Kivshar for the valuable discussions and hospitality during his stay at ANU and acknowledges the support of the Polish Government Research Grant for 2006-2009. N. V. H. was supported by Polish Ministry of Science and Education under grant N202 014 31. M.M. acknowledges support from the Foundation for Polish Science and ARC Center of Excellence for Quantum Atom Optics.
[1] V. E. Zakharov and A. B. Shabat, Sov. Phys. JETP 34, 62 (1972).

[2] S. V. Dmitriev, D. A. Semagin, A. A. Sukhorukov, and T. Shigenari1, Phys. Rev. E 66, 046609 (2002).

[3] A. D. Martin, C. S. Adams, and S. A. Gardiner, Phys. Rev. Lett. 98, 020402 (2007).

[4] G. I. Stegeman and M. Segev, Science 286, 1518 (1999).

[5] D. Andersen and M. Lisak, Phys. Rev. A 32, 2270 (1995).

[6] P. Chamorro-Posada and G. S. McDonald, Phys Rev. E 74, 036609 (2006).

[7] H. E. Nistazakis, D. J. Frantzeskakis and B. A. Malomed,
Phys. Rev. E, 64, 026604 (2001).

[8] L. Khaykovich, F. Schreck, G. Ferrari, T. Bourdel, J. Cubizolles, L. D. Carr, Y. Castin, and C. Salomon, Science 296, 1290 (2002); K. E. Strecker, G. B. Partridge, A. G. Truscott and R. G. Hulet, Nature 417, 150 (2002); V. M. Pérez-García, H. Michinel, and H. Herrero, Phys. Rev. A 57, 3837 (1998).

[9] L. D. Carr, J. Brand, Phys. Rev. A 70, 033607 (2004); M. I. Rodas-Verde, H. Michinel, V. M. Perez-Garcia, Phys. Rev. Lett. 95, 153903 (2005); A. V. Carpentier, H. Michinel, Europhys. Lett 78, 10002 (2007). 
[10] L. Salasnich, A. Parola, and L. Reatto, Phys. Rev. A 65, 043614 (2001); ibid Phys. Rev. A 66, 043603 (2002).

[11] J. M. Gerton, D. Strekalov, I. Prodan, and R. G. Hulet, Nature 408, 692 (2000).

[12] E. A. Donley, N. R. Claussen, S. L. Cornish, J. L. Roberts, E. A. Cornell, and C. E. Wieman, Nature 412, 295 (2001).

[13] Q. Y. Li, C. Pask, R. A. Sammut, Opt. Lett. 16, 1083 (1991); R. A. Sammut and C. Pask, J. Opt. Soc. Am. B 8, 395 (1991).

[14] K.M. Hilligsøe, M.K. Oberthaler, and K.-P. Marzlin, Phys. Rev. A 66, 063605 (2002).

[15] L. Khaykovich and B. A. Malomed, Phys. Rev. A 74, 023607 (2006).

[16] O. Bttner, M. Bauer, S. O. Demokritov, B. Hillebrands, M. P. Kostylev, B. A. Kalinikos A. N. Slavin, Phys. Rev. Lett. 82, 4320 (1999).

[17] S. Raghavan, G. P. Agrawal, Opt. Commun. 180, 377 (2000).
[18] R. Y. Chiao, E. Germire and C. H. Townes, Phys. Rev. Lett 13, 479 (1964).

[19] B. A. Malomed, in: Progress in Optics, vol. 43, p. 71 (ed. by E. Wolf: North Holland, Amsterdam, 2002).

[20] V. M. Pérez-García, H. Michinel, J. I. Cirac, M. Lewenstein, and P. Zoller, Phys. Rev. Lett. 77, 5320 (1996).

[21] L. E. Sadler, J. M. Higbie, S. R. Leslie, M. Vengalattore, and D. M. Stamper-Kurn, Nature 443, 312 (2006).

[22] B. A. Malomed and S. Wabnitz, Opt. Lett. 16, 1388 (1991); G. Huang, M. G. Velarde, and V. A. Makarov, Phys. Rev. A 64, 013617 (2001); J. Babarro, M. J. PazAlonso, H. Michinel, J. R. Salgueiro, and D. N. Olivieri, Phys. Rev. A 71, 043608 (2005).

[23] M. Desaix, D. Anderson, M. Lisak, J. Opt. Soc. Am. B 8, 2082 (1991).

[24] J. Garnier, F. Kh. Abdullaev, B. B. Baizakov, Phys. Rev. A 69,053607 (2004). 